\title{
Mineraes da mina de Furnas Anglesita e Calamina
}

(5 figuras no texto)

\author{
R. Saldanha
}

Na mesma jazida de Furnas (municipic de Iporanga), de que estudamos a cerussita em trabalho anterior, verifica-se, entre outros mineraes secundarios, a existencia da anglesita e da calamina encontradas junto á galena argentifera apenas parcialmente alterada.

Nenhum dos que se dedicaram ao estudo dos veios de Furnas demorou-se na descripção morphologica dos crystaes que ali occorrem. A breve noticia dada por T KNECHT (1), registra unicamente certas formas da anglesita cuja determinação é aproximativa.

(1) T. KNECHT - Nota sobre alguns mineraes secundarios de chumbo da mina de Furnas, no Municipio de Iporanga - Bol. de Agricultura, S. Paulo, 1932. 


\section{A N GLE S I T A}

A anglesita apresenta-se geralmente na jazida com pequenas dimensões ( 1 a $5 \mathrm{~mm}$.), medindo, entretanto, mais de $2 \mathrm{~cm}$. segundo o eixo dos "z" quando se accentúa o habitus prismatico. São crystaes incolores e de boa transparencia, em agrupamentos irregulares que se dispõem nas cavidades do minerio. Muitos crystaes de cerussita pseudomorpha de anglesita são tambem encontrados, mesmo entre os de maior desenvolvimento.

As formas que determinamos são em numero de 13 e dentre ellas podemos chamar a atenção para $\{101\}$ que unicamente fôra assignalada por REDLICH (2) na anglesita de Josefistolen. Das nossas pesquisas resultou uma perfeita determinação da fórma, com absoluta segurança, o que nos pareceu util assignalar.

Damos a seguir a relação completa:

$$
\begin{aligned}
& \mathrm{a}\{100\} \quad \mathrm{m}\{110\}, \mathrm{n}\{120\} \\
& \mathrm{b}\{010\}, \quad \text { o }\{011\} \\
& \mathrm{c}\{001\}, \mathrm{s}\{101\}, \mathrm{d}\{102\} \\
& \mathrm{z}\{111\}, \quad \mathrm{r}\{112\}, \mathrm{y}\{122\}, \mathrm{u}\{123\}, \mathrm{i}\{144\}
\end{aligned}
$$

Dentre as medidas angulares constantes da tabella annexa esco-

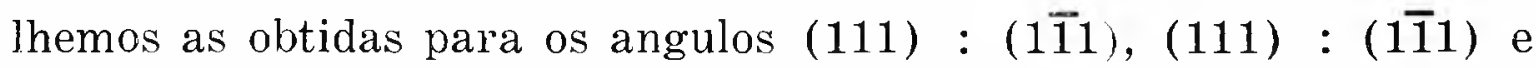
(111) : (110), nos crystaes que deram melhores medidas, com ellas estabelecendo a relação parametrica (os angulos calculados com estas constantes são dadas na sexta columna da tabella) :

$$
\mathrm{a}: \mathrm{b}: \mathrm{c}=0.7766: 1: 1,2153
$$

Emquanto para o parametro "a" obtivemos valor aproximado dos que foram determinados em material de outras jazidas, para o parametro " $c$ " encontramos um valor minimo, com forte diferença 1900 .

(2) K. A. REdich - Ref. Zt. f. Krystallographie - 32 Bd., 182, $183-$ 


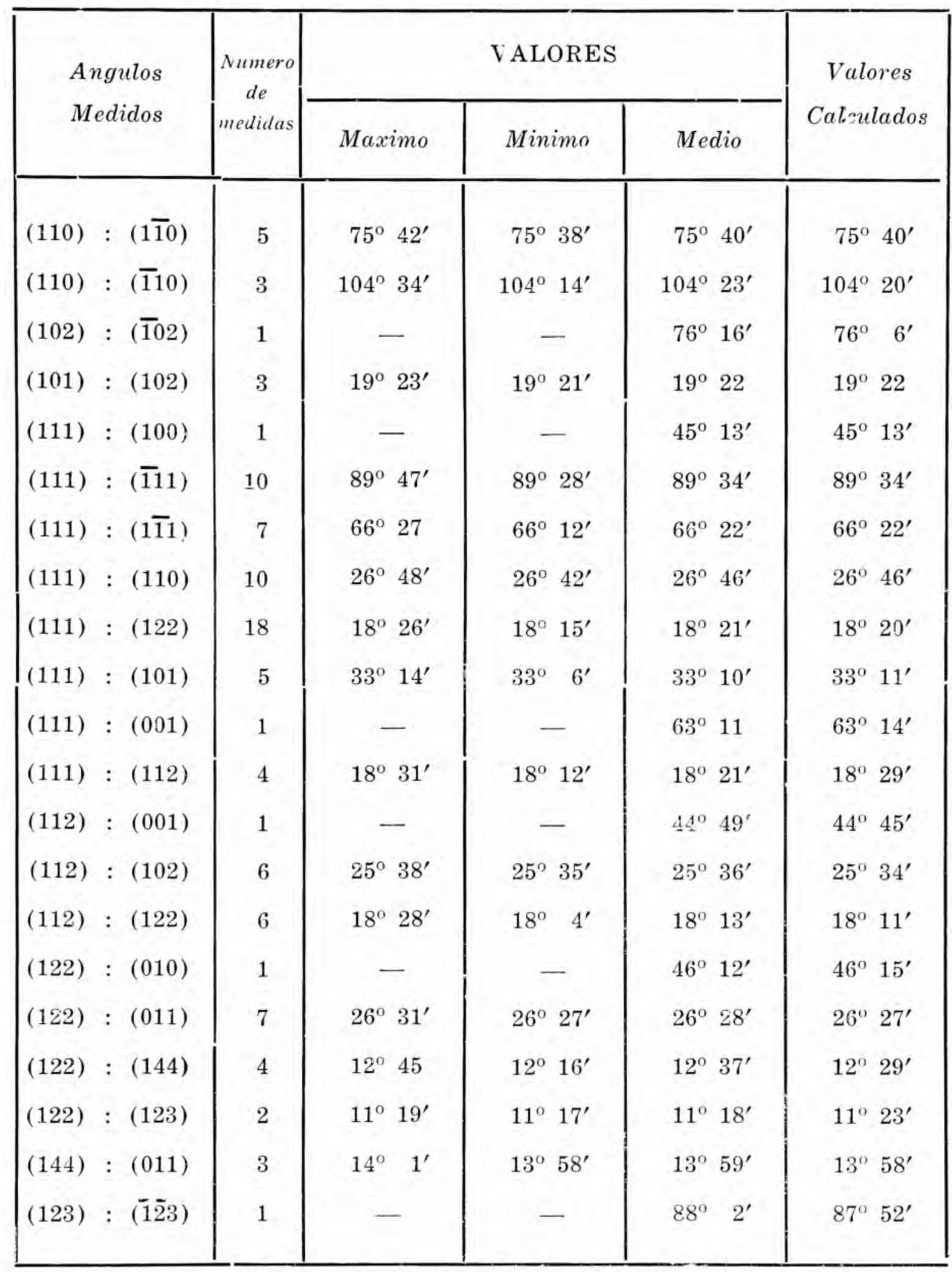


na segunda casa decimal. São estas as relações encontradas por alguns outros autores:

$$
\begin{aligned}
\mathrm{a}: \mathrm{b} & : \mathrm{c} \\
078516: 1 & : 1.28939 \text { Kokscharow } \\
07841: 1 & : 1.2806 \text { Hauy } \\
07837: 1 & : 1.2867 \text { Kupffer } \\
0.7928: 1 & : 1.3017 \text { Mohs } \\
0.8038: 1 & : 1.2904 \text { Philipps } \\
0.7865: 1 & : 1.2910 \text { Dana } \\
0.7865: 1 & : 1.2923 \text { Miller _ Brooke }(3) .
\end{aligned}
$$

Escrevemos abaixo as principais combinações existentes na anglesita de Furnas.

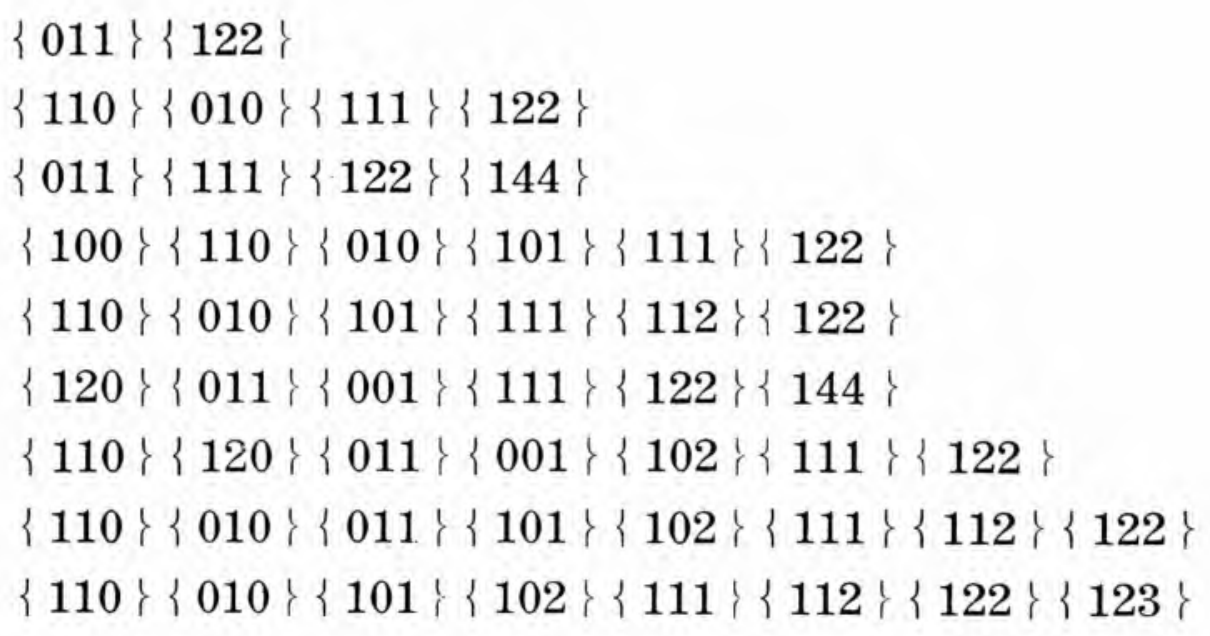

Persistindo, embora, em todas ellas, a pyramide $\{122\}$ o habitus é variado, ora com o maior desenvolvimento de $\{110\}$, ora (e com maior frequencia) com a predominancia das formas $\{\mathrm{hkl}\}$

O habitus prismatico é sempre terminado pelas pyramides e muitas vêzes acompanhado pelo prisma horizontal $\{101\}$ e pelos pinacoides $\{100\}$ e $\{010\}$, todos pouco desenvolvidos (fig. 1).

O habitus pyramidal caracterisa-se pelo maior desenvolvimento de $\{111\}$, tambem nelle existindo outras fórmas, inclusive prismas

(3) Handbuch der Mineralogie (C. Hintze) - 1Bd, 3Ab. 3962. 
horizontaes e verticaes (fig. 2). Alguns exemplares se distinguem pela riqueza de fórmas e bellos reflexos das mesmas.

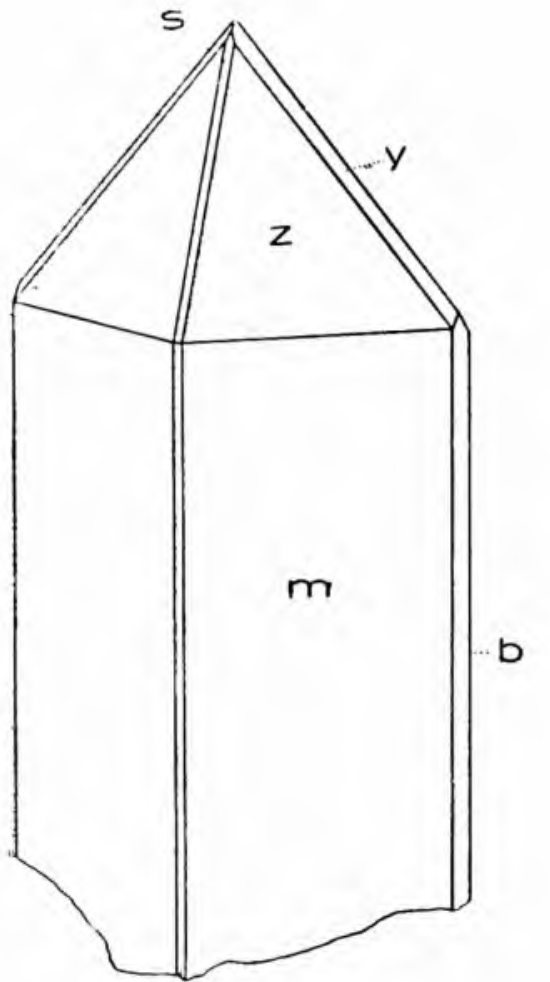

a

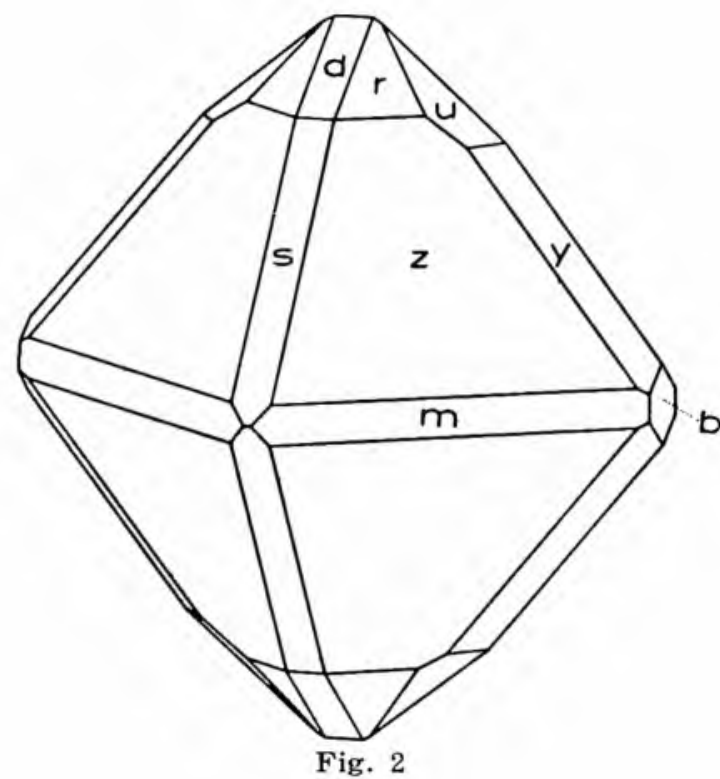

Fig. 1

Um outro habitus chama a atenção pelo aspecto original de que se reveste. Nelle se destacam as zonas [011] e [011], com a combinação das pyramides $\{111\},\{122\}$ e $\{144\}$ com $\rho$ prisma $\{011\}$ (fig. 3).

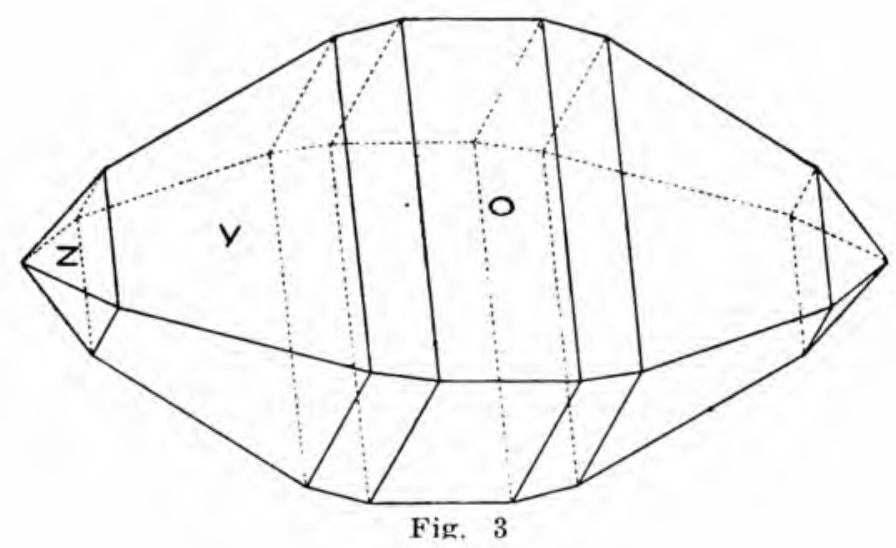

Entre os pinacoides o mais frequente é $\{010\}$, mas tanto este como $\{100\}$ e $\{001\}$ são pequenas facetas que dão reflexos fracos ao goniometro. 
As faces do prisma $\{110\}$, frequentes e bem desenvolvidas, não são perfeitas. O prisma $\{120\}$ é raro e de pouco desenvolvimento.

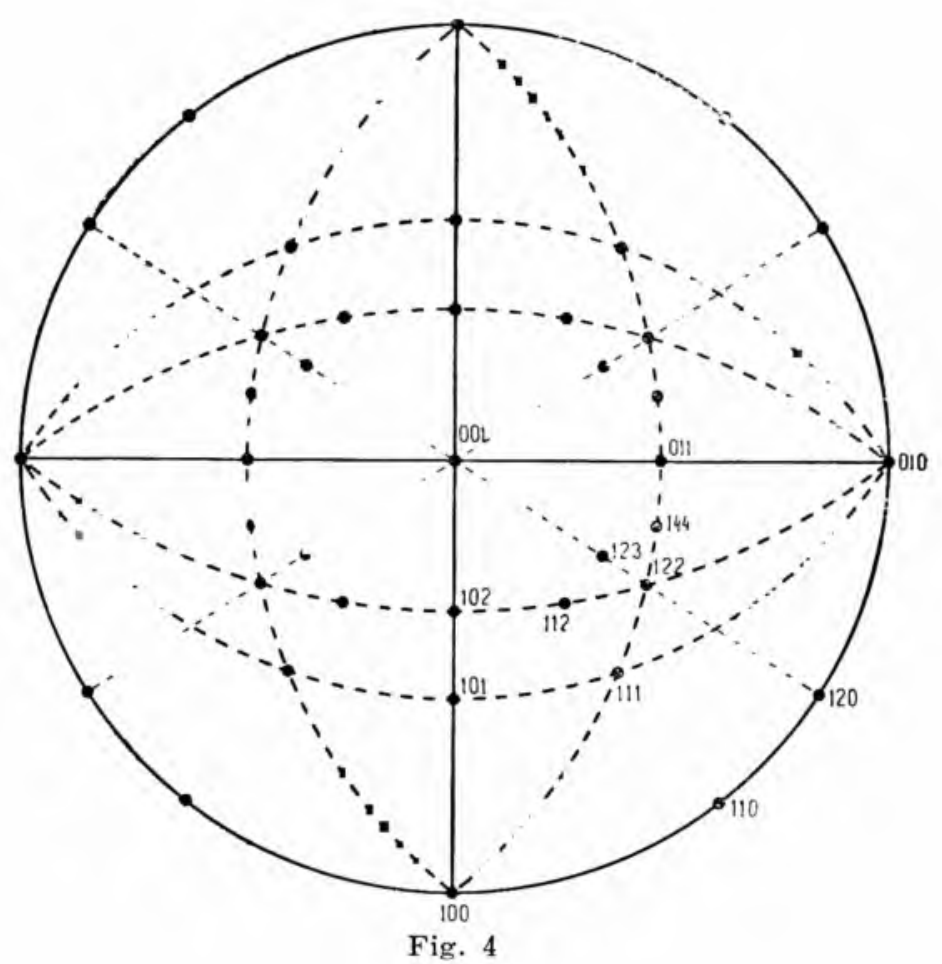

$\mathrm{Na}$ zona dos " $\mathrm{x}$ " apenas o prisma horizontal é encontrado em bellas faces. Na zona dos "y" temos $\{101\}$ e $\{102\}$, em fórmas finas e alongadas, dando bôas medidas.

Quanto ás pyramides verificamos, em çuasi todos os exemplares, a predominancia de $\{111\}$, especialmente no habitus pyramidal. A fórma $\{122\}$ é, entretanto, de uma constancia notavel no seu aparecimento, sendo mesmo a unica que observamos em todos os exemplares. A fórma $\{144\}$, a mais para rara na anglesita de Furnas, dá tambem, quando se desenvolve, faces de bons reflexos e de bôas proporções. As demais, $\{112\}$ e $\{123\}$, são na maior parte das vezes, de muito menores dimensões. 


\section{A L A M I N A}

Os crystaes da calamina de Furnas são, em regra, de 2 a $4 \mathrm{~mm}$. na maior dimensão, tabulares segundo $\{010\}$ Encontram-se em bellos agrupamentos, dispostos em rosetas, ou dispersos entre os crystaes de anglesita e com estes se confundindo por incolores e bem transparentes.

O habitus, em todos os exemplares observados, é notavel pela sua constancia. As fórmas encontradas são em pequeno numero:

$$
\begin{aligned}
& \mathrm{m}\{110\} \\
& \mathrm{b}\{010\}, \mathrm{u}\{011\}, \mathrm{i}\{031\} \\
& \mathrm{c}\{001\} \mathrm{s}\{101\}, \mathrm{t}\{301\} \\
& \mathrm{o}\{112\} ?
\end{aligned}
$$

Poucas são as combinações cuja existencia verificamos:

$$
\begin{aligned}
& \{110\},\{010\} \quad\{011\},\{001\},\{101\},\{301\}, \\
& \{110\},\{010\} \quad\{011\},\{031\},\{001\},\{101\},\{301\}, \\
& \{110\},\{010\},\{011\},\{031\},\{001\},\{101\},\{301\},\{112\} ?
\end{aligned}
$$

Poderiamos mesmo reduzil-as á segunda (fig. 5), ora sem a presença de $\{031\}$, mais raramente enriquecida com a occurrencia de $\{112\}$

O pinacoiảe $\{010\}$ dá-nos as faces de maior desenvolvimento, fortemente estriadas parallelamente a [100], emprestando ao crystal o seu habitus caracteristico. 
O pedion $\{001\}$ é fórma sempre presente, em pequenas facetas alongadas. O prisma $\{110\}$ e os domas $\{011\},\{101\}$ e $\{301\}$, tem todos faces perfeitas e de bôas proporções, com excellentes medidas ao goniometro. O doma $\{031\}$, em facetas muito pequenas, é, por vezes, de determinação apenas aproximativa.

A pyramide $\{112\}$ damol-a sob fórma interrogativa por náo termos obtido bons reflexos que permittissem medir com segurança os angulos com $\{011\}$ e $\{101\}$

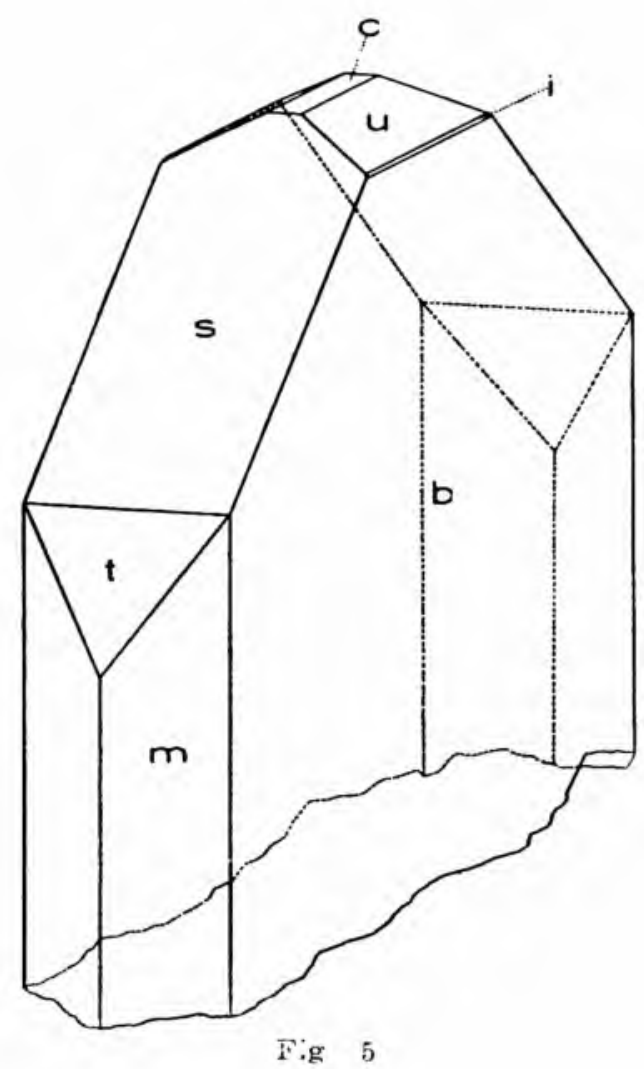

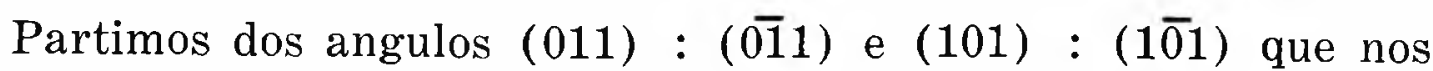
garantiam maior precisão para determinar a relação parametrica. A nossa é diversa das de Schrauf e E. Billows para a calamina de Altenberg e Ghergur, respectivamente. 
SCHRAUF (4) - 0,78340: $1: 0,47782$;

BILlOWS (5) - 0,78897: $1: 0,48689$;

SALDANHA $-0,7812: 1: 0,4773$.

A tabella junto dá os valores medidos ao goniometro e os valores calculados com as constantes obtidas.

\begin{tabular}{|c|c|c|c|c|c|}
\hline \multirow{2}{*}{$\begin{array}{l}\text { Angulos } \\
\text { Medidos }\end{array}$} & \multirow{2}{*}{$\begin{array}{c}\text { Numero } \\
\text { de } \\
\text { medidas }\end{array}$} & \multicolumn{3}{|c|}{ VALORES } & \multirow{2}{*}{$\begin{array}{c}\text { Valores } \\
\text { Calculados }\end{array}$} \\
\hline & & Maximo & Minimo & Medio & \\
\hline$(110):(1 \overline{10})$ & 5 & $76^{\circ} 13^{\prime}$ & $76^{\circ} \quad 1 \frac{1}{2^{\prime}}$ & $75^{\circ} 58^{\prime}$ & $76^{\circ}$ \\
\hline$(011):(0 \overline{11})$ & 8 & $51^{\circ} \quad 3^{\prime}$ & $51^{\circ} \quad 1 / 2^{\prime}$ & $51^{\circ} \quad 2^{\prime}$ & $51^{\circ} \quad 2^{\prime}$ \\
\hline$(011):(031)$ & 4 & $29^{\circ} 43^{\prime}$ & $29^{\circ} 24^{\prime}$ & $29^{\circ} 33^{\prime}$ & $29^{\circ} 35^{\prime}$ \\
\hline$(031):(010)$ & 4 & $35^{\circ} \quad 6^{\prime}$ & $34^{\circ} 42^{\prime}$ & $34^{\circ} 55^{\prime}$ & $34^{\circ} 54^{\prime}$ \\
\hline$(101):(001)$ & 8 & $31^{\circ} 30^{\prime}$ & $31^{\circ} 24$ & $31^{\circ} 25^{1 / 2} 2^{\prime}$ & $31^{\circ} 25^{1 / 2}{ }^{\prime}$ \\
\hline$(301):(101)$ & 8 & $30^{\circ} \quad 4^{\prime}$ & $29^{\circ} \quad 48^{\prime}$ & $29^{\circ} 57^{\prime}$ & $29^{\circ} 55^{\prime}$ \\
\hline$(101):(110)$ & 5 & $65^{\circ} 47^{\prime}$ & $65^{\circ} 45^{\prime}$ & $65^{\circ} 46^{\prime}$ & $65^{\circ} 48^{\prime}$ \\
\hline$(101):(011)$ & 5 & $39^{\circ} 40^{1 / 2^{\prime}}$ & $39^{\circ} 37^{\prime}$ & $39^{\circ} 38^{\prime}$ & $39^{\circ} 37^{\prime}$ \\
\hline$(011):(110)$ & 2 & $74^{\circ} \quad 42^{\prime}$ & $74^{\circ} 36^{\prime}$ & $74^{\circ} 39^{\prime}$ & $74^{\circ} 35^{\prime}$ \\
\hline$(301):(110)$ & 5 & $46^{\circ} 25^{\prime}$ & $46^{\circ} 15^{\prime}$ & $46^{\circ} 171 / 2^{\prime}$ & $46^{\circ} \quad 181 / 2^{\prime}$ \\
\hline
\end{tabular}

Determinamos facilmente os indices de refracção recorrendo ás faces de domas. A angulo formado por (301) e (30̄1) serviu-nos para a medida de $n_{\alpha}$ e $n_{\gamma} ; o$ angulo $(010)$ e $(0 \overline{1} 1)$ para a de $n_{\beta}$.

(4) Schrauf - Handbuch der Mineralogie (Carl Hintze), 2 Bd, 1316, 1317.

(5) E. Billows - Riv. di Mineralogia e Crist. Italiana, 1908, Vol. 34, 
Servimo-nos da luz de $\mathrm{Na}$ e comparamos os resultados com os de outros autores.

\begin{tabular}{|c|c|c|c|c|c|c|}
\hline \multirow{2}{*}{ Autor } & \multirow{2}{*}{$\begin{array}{c}\text { Locali- } \\
\text { dade }\end{array}$} & \multicolumn{3}{|c|}{ Indices } & \multicolumn{2}{|c|}{ Ang. eixos opticos } \\
\hline & & $\mathrm{n}_{\alpha}$ & $n_{i}$ & $\mathrm{n}_{\gamma}$ & $\begin{array}{c}2 \mathrm{E}_{\mathrm{a}} \\
\text { (medido) }\end{array}$ & $\begin{array}{c}2 \mathrm{~V}_{\mathrm{a}} \\
\text { (calculado) }\end{array}$ \\
\hline V. V. Lang (6) & - & 1.61358 & 1.61696 & 1.63597 & $78^{\circ} 39^{\prime}$ & $46^{\circ} \quad 9^{\prime}$ \\
\hline Des Cloiseaux (7) & Figeac & 1.615 & 1.618 & 1.635 & $78^{\circ} 20^{\prime}$ & $45^{\circ} 57^{\prime}$ \\
\hline E. Billows (8) & Ghergur & 1.6138 & 1.6178 & 1.6358 & $48^{\circ} \quad 48^{\prime}(9)$ & $51^{\circ} 28$ \\
\hline U. Panichi $(10)$ & Bleiberg & 1.61376 & 1.61673 & 1.6355 & - & - \\
\hline R. Saldanha & Furnas & 1.6132 & 1.6166 & 1.6355 & $78^{\circ} 14^{\prime}$ & $45^{\circ} 56^{\prime}$ \\
\hline
\end{tabular}

Para a mesma luz, e como media de quatro medidas, determinamos como angulo aparente dos eixos opticos no ar:

$$
2 \mathrm{E}_{\mathrm{a}}=78^{\circ} 14^{\prime}
$$

calculando o angulo verdadeiro:

$$
2 \mathrm{~V}_{\mathrm{a}}=45^{\circ} 56^{\prime}
$$

(6) V. v. LANG - Handbuch der Mineralogie (Carl Hintze), 2Bd., 1314.

(7) Des Cloiseaux - Idem, idem, 1314, 1323.

(8) E. Billows - Obra citada.

(9) A determinação do angulo aparente foi feita por Billows com im. mersão cm liquido de Thoulet, de indice de refracção 1.7004 .

(10) V. PANichi - Rf. 2t. f. Krystallographie, 44 Bd., 620. 


\title{
SUMMARIUM
}

\begin{abstract}
ANGLESITA
Anglesita e Furnas nullius coloris crystallis apparet, ex uno ad quinque amplitudine millimetra cum piramidato. ad quinque dimidias centimetri partes cum prismatico afficiatur habitu.

Quas reperimus formas: a $\{100\}, \mathrm{m}\{110\}, \mathrm{n}\{120\}, \mathrm{b}\{010\}$ o $\{011\} \mathrm{c}\{001\}, \mathrm{s}\{101\}, \mathrm{d}\{102\}, \mathrm{z}\{111\}, \mathrm{r}\{112\}, \mathrm{y}\{122\}$, $\mathrm{u}\{123\}, \mathrm{i}\{144\}$
\end{abstract}

Forma $\{101\}$ tantum in Anglesita e Josefistolo reperta.

Relatio parametrica : $\mathrm{a}: \mathrm{b}: \mathrm{c}=0,7766: 1: 1,2153$

\section{CALAMINA}

Calamina e Furnas tabularis apparet secundum $\{010\}$ parvis ex duobus ad quattuor amplitudinis millimetra crystallis.

Quas reperimus formas : $\mathrm{m}\{110\}, \mathrm{b}\{010\}, \mathrm{u}\{011\}, \mathrm{i}\{031\}$, $\mathrm{c}\{001\}, \mathrm{s}\{101\}, \mathrm{t}\{301\}, \mathrm{o}\{112\}$ ?

Relatio parametrica : $\mathrm{a}: \mathrm{b}: \mathrm{c}=0,7812: 1: 0,4773$

Refractionis index : $n_{\alpha}=1,6132 n_{\gamma}=1,6166 n_{j}=1,6355$

Opticorum axium angulus : $2 \mathrm{E}_{\mathrm{a}}=78^{\circ} 14^{\prime} \quad 2 \mathrm{~V}_{\mathrm{a}}=45^{\circ} 56^{\prime}$

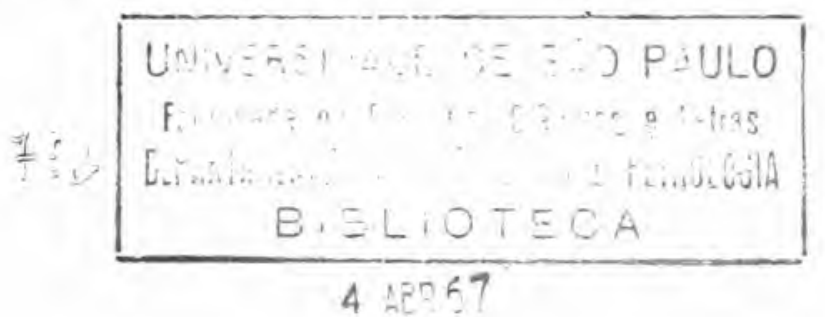

